\title{
Welcome to Hydrogen-A New International and Interdisciplinary Open Access Journal of Growing Interest in Our Society
}

\author{
George E. Marnellos 1,2,*(D) and Thomas Klassen ${ }^{3,4, *(D)}$ \\ 1 Department of Mechanical Engineering, University of Western Macedonia, 50100 Kozani, Greece \\ 2 Chemical Process and Energy Resources Institute, Centre for Research \& Technology Hellas, Thermi, \\ 57001 Thessaloniki, Greece \\ 3 Institute of Hydrogen Technology, Helmholtz-Zentrum Geesthacht, Center for Materials and Coastal \\ Research, 21502 Geesthacht, Germany \\ 4 Materials Technology, Helmut Schmidt University, University of the Federal Armed Forces Hamburg, \\ 22043 Hamburg, Germany \\ * Correspondence: gmarnellos@uowm.gr (G.E.M.); klassen@hsu-hh.de (T.K.)
}

Received: 21 December 2020; Accepted: 21 December 2020; Published: 22 December 2020

The 2018 Intergovernmental Panel on Climate Change (IPCC) report [1] highlighted the need to significantly reduce anthropogenic greenhouse gas (GHG) emissions to effectively address the threat to our planet from climate change. Emissions should drop by at least 45 percent by 2030 compared to their 2010 levels and to net-zero by 2050 if we aim to stay below +1.5 degrees Celsius of global warming. The alternative is daunting, according to Doomsday Clock [2], a tipping point could soon be passed, and global warming would accelerate by self-reinforcing feedback processes. Then, more severe climate phenomena and major habitat losses would push the planet closer to the possibility of a man-made disaster.

In this sense, several public groups, many young activists, scientists, and concerned consumers, are calling for more ambitious steps to reduce carbon emissions. By setting aggressive decarbonization goals, intergovernmental organizations and most world leaders are now responding to this message. During the 2019 UN Climate Summit, 66 countries announced their intention to meet zero carbon emissions goals by 2050. The European Union legislation includes possible sanctions for failure to meet the targets, and the Green Deal strategic plan to support the carbon-neutrality target has been initiated. In the U.S., 25 states have formed a Climate Alliance, collectively committed to reducing GHG emissions by at least 26-28\% in 2025 as compared to 2005 [3]. Finally, China has revised its climate policy targets to meet peak emissions by 2030 and to achieve its goal of $20 \%$ of non-fossil fuel primary energy demand by investing in sustainable technologies [4].

Hydrogen is the lightest and most abundant chemical element in nature and the cornerstone in Mendeleyev's periodic table. Hydrogen is now the focus of many key actors in the academic and research community as well as in industrial and political arenas to leverage and facilitate our energy transition from fossil fuels to renewables.

Hydrogen represents a versatile, scalable, and flexible energy vector for our future sustainable energy supply. Although hydrogen is not an energy source itself, it offers many advantages in unlocking the large-scale incorporation of renewables in the energy mix compared to other technologies, because $t$ allows energy to be efficiently transformed, processed, and stored. Hydrogen can be used as a buffer for renewables and for energy delivery across various sectors of the economy and geographical regions.

Hydrogen is particularly needed to replace natural gas: our gas grid supports industry, delivers heat to households, and is currently primarily used for power generation. Hydrogen alternatives suffer either scale-up problems as in the case of biogas or require costly building retrofits as with the electrification 
with heat pumps. Batteries contain scarce elements and are too costly for large scale energy storage. Furthermore, the electric grid has limited capacity and upgrading all power installations to higher power is very expensive. Without the need for significant upgrades, hydrogen can be introduced as natural gas-hydrogen blends through the current gas pipeline network and gradually convert gas grids to operate on pure hydrogen or even synthetic natural gas (SNG) derived from electrolytic renewable hydrogen and recycled industrial $\mathrm{CO}_{2}$ emissions.

Hydrogen is also the most promising decarbonization option in the mobility and transport sector, i.e., for trucks, buses, ships, trains, large cars, and commercial vehicles, where low energy density, high initial costs, and the slow recharge performance of batteries are major drawbacks.

In addition, industry can utilize hydrogen to produce high-quality heat or use it as a raw material in several processes, either directly or together with captured $\mathrm{CO}_{2}$ as synthetic hydrocarbons, i.e., synfuel or electro-fuel. For example, in the steel industry, $\mathrm{H}_{2}$ can function as a reductant to replace coal-based blast furnaces. In the future, low-carbon sources could supply the hydrogen required for ammonia synthesis and hydrotreating in refineries. Hydrogen, along with $\mathrm{CO}_{2}$, can also displace fossil hydrocarbons in chemical processes, such as the manufacture of olefins and hydrocarbon solvents (BTX), which are the fundamental building blocks for several every-day goods, also acting as a carbon sink where $\mathrm{CO}_{2}$ is used instead of emitted.

This vision for a hydrogen economy, which was first introduced as a term by John Bockris during a talk he gave in 1970 at General Motors (GM) Technical Center [5], is foreseen to play an integral role in the transition to renewable energy sources by providing a mechanism to flexibly transfer energy across sectors, time, and place. The energy transition requires almost completely decarbonized power generation, which implies the need to integrate renewables into the grid. Sector coupling is the ultimate goal of an energy vector, and hydrogen is the only solution available with the scale-up potential to convert generated power into a form required by end-users, to store it, and distribute it on demand.

The electrification of society needs greater amounts of energy from variable renewables, therefore imbalances in supply/demand for both short and long periods will increase. This generates the need for improved year-round balance and seasonal energy storage. Although short-term stability can indeed be provided by batteries and smart grids, hydrogen is the only at-scale technology available for long-term energy storage. It can use current gas grids, salt caverns, and depleted gas fields to store energy at a low cost for longer periods.

Hydrogen also provides a connection between low-cost renewables and demand-centered regions. Hydrogen enables energy transport in pipelines, ships, or trucks over long distances, whether gaseous, liquified, or bonded to other elements or molecules, at a much lower cost than power transmission lines.

Hydrogen as a core fuel will cover a substantial part of the energy needs and services in our future energy economy. However, large-scale deployment of hydrogen across the globe is still facing several challenges. Further improvements and more innovative and economically competitive solutions in hydrogen production, storage, transport, and use are needed. Hence there is plenty of ground for researchers and engineers to optimize efficiencies, explore new families of materials (with emphasis on abundant and non-toxic ones), identify novel devices and integrated processes, improve lifetimes, and substantially decrease the costs for scaling up the hydrogen technologies.

In this very sense, we are pleased to welcome you to the latest international, interdisciplinary, and exciting open-access journal Hydrogen.

Hydrogen aims to be an advanced forum for scientists and engineers worldwide to share, promote and disseminate their fundamental discoveries and research innovations in the field of hydrogen science and technology as well as their studies regarding the market and socio-economic prospects of Hydrogen economy. The topics of interest include (but are not limited to):

- Hydrogen generation: reforming and partial oxidation of fossil and bio-based fuels; low and high temperature atmospheric and pressurized electrolysis of water (and $\mathrm{CO}_{2}$ ); thermochemical (e.g., gasification, pyrolysis) or biological (e.g., anaerobic digestion, fermentation) conversion of solid fuels; photo-electrochemical water splitting; thermochemical cycles; hydrogen production and 
separation in membrane reactors using hydrocarbons or hydrogen-containing compounds (e.g., $\mathrm{H}_{2} \mathrm{~S}$ ) as feedstock.

- Hydrogen storage: cryogenic; compressed; material-based storage (e.g., metal hydrides or other hydrides); chemical storage; liquid organic hydrogen carriers; underground storage.

- Hydrogen transport, distribution, and infrastructure: transport by trucks, trains, and ships; transportation and distribution through natural gas pipeline networks.

- Hydrogen use: as a fuel for power generation in thermal engines and fuel cells; as fuel in cars, buses, vans, light and heavy-duty trucks, trains, and in the maritime and aviation sectors; as feedstock in the chemical sector, in refineries and steel industry.

- Reactions with hydrogen: hydrogenation (e.g., ammonia and methanol synthesis, hydrotreating reactions, metal hydrides) and dehydrogenation (e.g., alkanes to alkenes, dehydrocyclization, recovery of hydrogen by hydrocarbons off-streams) reactions; hydrogenation of captured $\mathrm{CO}_{2}$ emissions to chemicals and fuels (e.g., synthetic natural gas and liquid fuels through Fischer-Tropsch process), catalysis of hydrogen reactions.

- Hydrogen applications: feasibility assessment and life cycle analysis studies of integrated hydrogen technologies; hydrogen safety aspects; codes, standards, and norms; regulation aspects; social acceptance and awareness issues; market studies and prospects; large deployment infrastructures; national roadmaps.

- Fundamental aspects: thermodynamics; properties; isotopes; compounds; phases; atomic and molecular hydrogen.

Hydrogen publishes timely and original research, systematic reviews, and short communications on all branches of hydrogen science and technology. There is no limitation on the length of the articles, and it is important to include complete information (experimental, modeling, or otherwise), so that the findings can be replicated.

By publishing certain topical issues of special interest, we aim for a great start to the journal in its first year. For issues that should be covered in a special issue, we welcome your suggestions for suitable guest editors.

We hope to receive your finest work for publication in this journal and welcome your comments and suggestions on how to make Hydrogen an exceptional journal.

\section{References}

1. Special Report: Global Warming of $1.5^{\circ} \mathrm{C}$; Intergovernmental Panel on Climate Change: Geneva, Switzerland, 2018.

2. Mecklin, J. It is 100 seconds to midnight. In 2020 Doomsday Clock Statement; Bulletin of the Atomic Scientists: Chicago, IL, USA, 2020.

3. 2019 Fact Sheet; United States Climate Alliance: Washington, DC, USA, 2019.

4. Qi, Y.; Stern, N.; He, J.; Lu, J.; King, D.; Liu, T.; Wu, T. China's peaking emissions and the future of global climate policy. In China's Energy in Transition Series; Brookings-Tsinghua Center for Public Policy: Beijing, China, 2018.

5. National Hydrogen Association. The History of Hydrogen; United States Department of Energy: Washington, DC, USA, 1970.

Publisher's Note: MDPI stays neutral with regard to jurisdictional claims in published maps and institutional affiliations.

(C) 2020 by the authors. Licensee MDPI, Basel, Switzerland. This article is an open access article distributed under the terms and conditions of the Creative Commons Attribution (CC BY) license (http://creativecommons.org/licenses/by/4.0/). 